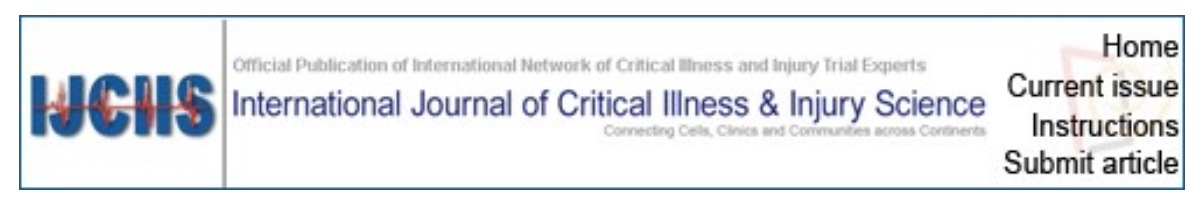

Int J Crit IIIn Inj Sci. 2018 Jan-Mar; 8(1): 36-40.

PMCID: PMC5869798

doi: $10.4103 /$ IJCIIS.IJCIIS_40_17

PMID: 29619338

\title{
Three- versus four-factor prothrombin complex concentrate for the reversal of warfarin-induced bleeding
}

\author{
Tara Holt, Scott Taylor, ${ }^{1}$ Prasad Abraham, ${ }^{2}$ Wesley Mcmillian, ${ }^{3}$ Serena Harris, ${ }^{4} \underline{\text { James Curtis }}^{5}$ and $^{\text {Tai Elder }^{1}}$ \\ Department of Pharmacy, IU Health Methodist Hospital, Indianapolis, IN, USA \\ ${ }^{1}$ Department of Pharmacy, Via Christi Hospitals, Wichita, KS, USA \\ 2 Department of Pharmacy, Grady Health System, Atlanta, GA, USA \\ ${ }^{3}$ Department of Pharmacy, Fletcher Allen Health Care, Burlington, VT, USA \\ ${ }^{4}$ Department of Pharmacy, Eskenazi Health, Indianapolis, IN, USA \\ ${ }^{5}$ Department of Pharmacy, Bronson Methodist Hospital, Kalamazoo, MI, USA
}

Address for correspondence: Dr. Tara Holt, Department of Pharmacy, IU Health Methodist Hospital, 1701 N. Senate BIvd, AG 401, Indianapolis, IN 46202, USA. E-mail: tholt4@iuhealth.org

Copyright : @ 2018 International Journal of Critical IIIness and Injury Science

This is an open access article distributed under the terms of the Creative Commons Attribution-NonCommercial-ShareAlike 3.0 License, which allows others to remix, tweak, and build upon the work non-commercially, as long as the author is credited and the new creations are licensed under the identical terms.

\section{Objective:}

Go to:

The objective of this study was to evaluate the effectiveness of 3-factor prothrombin complex concentrate (3F-PCC) compared to 4-factor PCC (4F-PCC) in warfarin-associated bleeding.

\section{Methods:}

Go to:

This multicenter, retrospective, cohort study analyzed data from patients admitted between May 2011 and October 2014 who received PCC for warfarin-associated bleeding. The primary outcome was the rate of international normalized ratio (INR) normalization, defined as an INR $\leq 1.3$, after administration of $3 \mathrm{~F}-\mathrm{PCC}$ compared to 4F-PCC. Other variables of interest included the incidence of additional reversal agents, new thromboembolic events, and mortality.

\section{Results:}

A total of 134 patients were included in the analysis. The average dose of PCC administered was $24.6 \pm 9.3$ units $/ \mathrm{kg}$ versus $36.3 \pm 12.8$ units $/ \mathrm{kg}$ in the $3 \mathrm{~F}-\mathrm{PCC}$ and $4 \mathrm{~F}-\mathrm{PCC}$ groups, respectively, $P<0.001$. Baseline INR in the 3F-PCC and 4F-PCC groups was $3.61 \pm 2.3$ and $6.87 \pm 2.3$, respectively $P<0.001$. 4F-PCC had a higher rate of INR normalization at first INR check post-PCC administration compared to 3F-PCC ( $84.2 \%$ vs. $51.9 \%, P=0.0001)$. Thromboembolic events, intensive care unit and hospital length of stay, and mortality were similar among both groups.

\section{Conclusion:}

Go to:

The use of 4F-PCC leads to a more significant reduction in INR compared to 3F-PCC though no difference in mortality or length of stay was observed. Thromboembolism rates were similar among both groups. 
Key Words: 3-factor prothrombin complex concentrate 4-factor prothrombin complex concentrate, anticoagulation reversal, emergent bleeding, prothrombin complex concentrate, warfarin reversal

Warfarin is a commonly used anticoagulant for the treatment of a variety of indications, most commonly deep vein thromboembolism, pulmonary embolism, and atrial fibrillation. Because warfarin has a narrow therapeutic window and interindividual dosing variability, bleeding events are of high concern with the incidence of major bleeding events reported between $0 \%$ and 10\%.[1,2] Emergency medicine physicians are often the first to evaluate patients on warfarin presenting with major bleeding. They make crucial treatment decisions including both if and how to reverse the anticoagulation effects of warfarin. While the mainstay of therapy for most warfarin-associated bleeding events is Vitamin $\mathrm{K}$ and fresh frozen plasma (FFP), prothrombin complex concentrate (PCC) is often utilized in cases of major or life-threatening bleeding. $[\underline{3}, \underline{4}]$

Warfarin exerts its anticoagulant effect by inhibiting the Vitamin K-dependent clotting factors II, VII, IX, and X.[5] The administration of PCC negates the anticoagulant effects of warfarin by quickly replenishing these clotting factors. Before 2013, only 3-factor PCC (3F-PCC) was available in the United States (US). Four-factor PCC (4F-PCC) has been available to providers in the US since May 2013 and is the only product to carry the Food and Drug Administration (FDA)-labeled indication of warfarin reversal. Both PCC formulations contain approximately 25 times the amount of factors II, IX, and X found in plasma. Four-factor PCC is unique in that it also contains ample amounts of factor VII.[3]

Despite the lack of an FDA approved indication for warfarin reversal, common practice in the US before 2013 had evolved from FFP to 3F-PCC for reversal of severe warfarin-associated bleeding, as well as bleeding from other types of oral anticoagulant therapy. After the FDA approval of 4F-PCC for urgent reversal of acquired coagulation factor deficiency induced by Vitamin K antagonist therapy (e.g., warfarin), many institutions and medical societies including the American College of Chest Physicians have begun to favor 4F-PCC.[6] Unfortunately, there are limited comparative data to determine what, if any, difference in safety or efficacy exists between PCC formulations. The purpose of this study is to evaluate the effectiveness of 3F-PCC compared to 4F-PCC in warfarin-associated bleeding.

\section{Study design and setting}

This was a multicenter, retrospective, cohort study conducted from May 2011 to September 2014 at five institutions in the US. All participating institutions received approval from their respective Investigational Review Boards. All patients receiving 3F-PCC or 4F-PCC for warfarin-associated bleeding within the study period were included in the analysis. Patients who were $<18$ years of age, pregnant, or prisoners were excluded from the study. Use of either 3F-PCC or 4F-PCC was based on each institution's medication formulary, therapeutic guideline, or provider discretion. All formulations were also dosed based on each institution's guideline, all of which round to the nearest vial size of the specific factor product. Baseline demographic information was collected and included age, sex, weight, indication for anticoagulation, baseline International normalized ratio (INR), bleed location, Acute Physiology and Chronic Health Evaluation II score,[7] and injury severity score for trauma admissions.[]]

\section{Outcomes}

The primary outcome was to compare the effectiveness of 3F-PCC versus 4F-PCC for the reversal of acute bleeding in patients on warfarin therapy. Effective reversal was defined as attainment of INR $\leq 1.3$. 
Secondary outcomes included the mean value and time of first INR post-PCC administration, mean INR value of any additional samples taken within $24 \mathrm{~h}$ post-PCC administration, incidence of additional anticoagulation reversal agents utilized, incidence of surgery within $12 \mathrm{~h}$ of PCC administration, and incidence of mortality. Safety was assessed by evaluation of the incidence of new thromboembolism within 7 days of PCC administration as determined by radiology report and/or physician documentation.

\section{Statistical analysis}

The sample size calculation was based on historical data with a predetermined alpha at 0.05 and beta of 0.8 . To reach significance, a total of 266 patients (133 per group) were needed to be analyzed. Data was presented in percentage or mean \pm standard deviation. Dichotomous data were compared using Chi-square or Fisher's exact test. All continuous data were normally distributed and analyzed with the Student's $t$-test.

RESULTS Go to:

\section{Characteristics of study subjects}

A total of 134 patients met study inclusion. Of those included, 77 (57.5\%) received 3F-PCC and 57 $(42.5 \%)$ received 4F-PCC. Overall, baseline characteristics were similar among the 3F-PCC and 4F-PCC groups [Table 1], with the exception of age (74 vs. 57 years; $P=0.013)$ and baseline INR (3.6 vs. $6.8 ; P<$ 0.001 ). In both groups, the main indications for anticoagulation were atrial fibrillation and treatment of venous thromboembolic disease.

\section{Main results}

Of the patients receiving 4F-PCC, 84.2\% achieved an INR $\leq 1.3$ compared to $51.9 \%(P<0.001)$ in those receiving 3F-PCC. Over $90 \%$ of patients in both groups received Vitamin $\mathrm{K}$ in addition to PCC $(96.5 \% 4 \mathrm{~F}-$ PCC vs. $90.9 \%$ 3F-PCC). The mean time to initial INR after PCC administration was $3.3 \pm 0.10 \mathrm{~h}$ in the $4 \mathrm{~F}-\mathrm{PCC}$ group versus $3.8 \pm 0.12 \mathrm{~h}$ in the $3 \mathrm{~F}-\mathrm{PCC}$ group $(P=0.32)$. The mean initial INR after 4F-PCC administration was $1.25 \pm 0.33$ and $1.40 \pm 0.27$ after 3F-PCC $(P=0.009)$. In those with additional INR values obtained within $24 \mathrm{~h}$ of administration, $78 \%$ of patients who received $4 \mathrm{~F}-\mathrm{PCC}$ and $73 \%$ who received 3F-PCC reached an INR $\leq 1.3$. Mean INR in those with additional INR values was $1.23 \pm 0.42$ with 4F-PCC and $1.25 \pm 0.18$ with 3F-PCC, which did not meet statistical significance $(P=0.79)$.

The mean PCC dose was $36.3 \pm 12.8$ units $/ \mathrm{kg}$ for patients receiving 4F-PCC and $24.6 \pm 9.3$ units $/ \mathrm{kg}$ for those receiving $3 \mathrm{~F}-\mathrm{PCC}$. The extent of INR reversal between the two groups broken down by PCC dose can be found in Table 2. The majority of patients in both groups received intravenous phytonadione (87.7\% 4F-PCC vs. 96.7\% 3F-PCC). Patients with a baseline INR $>4$ were more likely to reach goal INR in the 4FPCC group compared to 3F-PCC ( $84.4 \%$ vs. $40 \%$; $P=0.003)$. Similarly, more patients receiving 4F-PCC reached goal INR when baseline INR was $<4(84.0 \%$ vs. $56.1 \% ; P=0.03)$. Three patients received an additional dose of PCC in the 3F-PCC group and one patient received Factor VII in the 4F-PCC group. FFP was given to $17.5 \%$ of patients receiving $4-\mathrm{PCC}$ and $51.9 \%$ in those receiving $3-\mathrm{PCC}(P \leq 0.01)$. When excluding patients who received FFP, the results were similar to the overall findings. Of those receiving 4FPCC but no FFP, $82.9 \%$ achieved an INR $\leq 1.3$ compared to $56.7 \%$ in those receiving $3 F-P C C$ and no FFP $(P=0.01)$.

There was no difference in intensive care unit (5.8 vs. 6.7 days, $P=0.59)$ or hospital length of stay (9.0 vs. 11.6 days, $P=0.28)$, incidence of surgery within $12 \mathrm{~h}(80.7 \%$ vs. $84.2 \%, P=0.60)$, or mortality $(21.4 \%$ vs. $17.1 \%, P=0.65$ ) between the 4F-PCC and 3F-PCC groups. Two patients in each group developed a new thromboembolism within 7 days of $\mathrm{PCC}$ administration. 


\section{DISCUSSION}

In this study, 4F-PCC appears to be more effective at lowering INR to $\leq 1.3$ within $4 \mathrm{~h}$ of administration although this effect ultimately had no impact on length of stay or mortality. These results are similar to a single-center study of 53 patients conducted by Al-Majzoub et al. Their retrospective study noted a higher percentage of patients achieving INR reversal with approximately 26 units $/ \mathrm{kg}$ of 4F-PCC and 3F-PCC $(83.3 \%$ vs. $42.9 \%, P<0.01)$ with a similar length of stay and mortality rate.[9] Our study expands on these findings but provides increasing external validity by expanding the sample size and utilizing multiple centers, each with their own warfarin reversal protocols and dosing strategies.

When assessing efficacy of reversal agents for warfarin-associated bleeding, the ability of the intervention to reverse INR is often utilized as the primary outcome. As previously mentioned, warfarin affects multiple clotting factors, all with varying half-lives. Factor VII has the shortest plasma half-life $(5 \mathrm{~h})$ whereas the other affected clotting factors have half-lives between 15 and 65 h.[10] The INR is an imperfect marker of warfarin coagulopathy due to its differing sensitivity for the warfarin clotting factors. The INR assay measures activity of the extrinsic (factor VII) and common pathways (factor II and X) but not the intrinsic pathway (factor IX). Reagents used for the assay are most sensitive to factor VII so the presence or absence of factor VII will have the largest impact on the INR, whereas factor X and II will have a smaller impact, and factor IX no impact. The short half-life of factor VII, along with the variation of clotting factors found in PCC products can impact the INR. Using INR as an end point to assess efficacy of warfarin reversal is advantageous because it provides an objective endpoint, yet it has never been shown to correlate with improved outcomes.[11] While cessation of bleeding and improved clinical outcomes may be more beneficial outcomes, these are much harder to objectively measure and often depend on physician opinion, making them suboptimal for research studies.

In this study, an INR goal of 1.3 or less was used to determine the effectiveness of the reversal agents. An INR value of 1.3 was chosen based on previous PCC studies.[9,12] One can argue using an INR of 1.3 is an arbitrary goal and many surgeons often use a higher INR threshold of $<1.5$ or 1.7 when determining whether or not to perform a procedure. This should be considered for any future studies looking at INR reversal in warfarin-associated bleeding. In the current study, an INR of $<1.7$ occurred in $94 \%$ receiving $4 \mathrm{~F}-\mathrm{PCC}$ and $87 \%$ receiving $3 \mathrm{~F}-\mathrm{PCC}(P=0.149)$. This INR target may be an acceptable indicator of successful reversal to most clinicians and may have been a more clinically relevant endpoint.

Another element to consider when comparing outcomes is the difference in dosing strategies between the two groups. The mean dose of 3F-PCC administered was more conservative than most dosing recommendations with many sources recommending closer to 35-50 units $/ \mathrm{kg}$. $[13,14]$ Of the 3F-PCC group dosed at 30 units $/ \mathrm{kg}$ or greater in our study, $64.7 \%$ had an INR $\leq 1.3$ compared to $44.2 \%$ of those receiving $<30$ units $/ \mathrm{kg}$. In addition to the $3 \mathrm{~F}-\mathrm{PCC}$ dose being on the lower end of the recommended dose, it was also 12 units/kg less on average than 4F-PCC which may have also had a clinical impact on outcomes.

Patients receiving 3F-PCC and 4F-PCC are at increased risk of thromboembolism from reversal of anticoagulation in a patient with an underlying hypercoagulability state and through supplementation of clotting factors that promote clot formation. The larger factor VII content of 4F-PCC compared to 3F-PCC has been theorized to put patients at higher risk for clot development. Our study showed similar rates of thromboembolism within each group. In patients receiving 3F-PCC, one patient developed a deep vein thrombosis, and one was found to have an upper subclavian clot. In those receiving 4F-PCC, one patient had an ischemic stroke and one developed a deep vein thrombosis.

There is a significant cost increase associated with 4F-PCC compared to 3F-PCC. When determining formulary status of medications at an institution, one must first focus on efficacy and safety and then, only if those aspects are deemed similar, cost must also be utilized in the decision of which agent to carry. 
Despite the lack of head-to-head trials comparing the two PCC formulations for anticoagulation reversal, many hospitals have switched their formulary agent to 4F-PCC because of its additional factor VII content and the theoretical benefit it encompasses. Because there are no prospective comparative trials, it is yet to be determined if the more rapid INR reversal observed with 4F-PCC truly improves clinical outcomes over $3 \mathrm{~F}-\mathrm{PCC}$ to justify the routine use of a more expensive agent with a potential for higher thrombotic events.

\section{Limitations}

While one strength of this study is that its external validity was increased because it was conducted at multiple, nonassociated institutions, this also provides limitations. Each institution has its own anticoagulation reversal guidelines that utilize different PCC dosing recommendations which could lead to varying strategies for reversal. There was no standardization in the time of INR collection after PCC administration though time of collection appeared similar among both groups. This study was also retrospective in nature which prevents the ability to standardize the reversal approach to ensure equivalent timing, dosing strategy, and concomitant therapies, whereas also requiring investigators to rely on historical charting for data collection. Despite the small number of patients and potential impact on power, the sample size was sufficient to demonstrate a significant difference in the primary outcome, achievement of target INR. As previously mentioned, INR has not been shown to correlate with improved clinical outcomes and may not be the best end point to determine the effectiveness of the reversal agents.

\section{CONCLUSION}

Go to:

Patients receiving 4F-PCC had a more significant reduction in INR when compared to 3F-PCC though this did not result in a decrease in the length of stay or mortality. The rate of thromboembolism was small and similar among both groups.

Financial support and sponsorship

Nil.

\section{Conflicts of interest}

There are no conflicts of interest.

REFERENCES

1. Levine MN, Raskob G, Landefeld S, Kearon C. Hemorrhagic complications of anticoagulant treatment. Chest. 2001;119:108S-21S. [PubMed: 11157645]

2. Wysowski DK, Nourjah P, Swartz L. Bleeding complications with warfarin use: A prevalent adverse effect resulting in regulatory action. Arch Intern Med. 2007;167:1414-9. [PubMed: 17620536]

3. Rodgers GM. Prothrombin complex concentrates in emergency bleeding disorders. Am J Hematol. 2012;87:898-902. [PubMed: 22648513]

4. Patanwala AE, Acquisto NM, Erstad BL. Prothrombin complex concentrate for critical bleeding. Ann Pharmacother. 2011;45:990-9. [PubMed: 21730276]

5. Warfarin [Package Insert] New Jersey: Bristol-Myers Squibb Company; 2011.

6. Guyatt GA, Akl EA, Crowther M, Gutterman DD, Schuunemann HJ. American college of chest physicians antithrombotic therapy and prevention of thrombosis panel. Executive summary. ACCP evidence-based clinical practice guidelines (9th edition) Chest. 2012;121(Suppl 2):7S, 14S.

[PMCID: PMC3278060] 
7. Knaus WA, Draper EA, Wagner DP, Zimmerman JE. APACHE II: A severity of disease classification system. Crit Care Med. 1985;13:818-29. [PubMed: 3928249]

8. Bolorunduro OB, Villegas C, Oyetunji TA, Haut ER, Stevens KA, Chang DC, et al. Validating the injury severity score (ISS) in different populations: ISS predicts mortality better among hispanics and females. J Surg Res. 2011;166:40-4. [PubMed: 20828742]

9. Al-Majzoub O, Rybak E, Reardon DP, Krause P, Connors JM. Evaluation of warfarin reversal with 4-factor prothrombin complex concentrate compared to 3-factor prothrombin complex concentrate at a tertiary academic medical center. J Emerg Med. 2016;50:7-13. [PubMed: 26433428]

10. Horton JD, Bushwick BM. Warfarin therapy: Evolving strategies in anticoagulation. Am Fam Physician. 1999;59:635-46. [PubMed: 10029789]

11. Voils SA, Baird B. Systematic review: 3-factor versus 4-factor prothrombin complex concentrate for warfarin reversal: Does it matter? Thromb Res. 2012;130:833-40. [PubMed: 23137921]

12. Pabinger J, Brenner B, Kalina U, Knaub S, Nagy A, Ostermann H. Prothrombin complex concentrate (Beriplex ${ }^{\circledR} \mathrm{P} / \mathrm{N}$ ) for emergency anticoagulation reversal: A prospective multinational clinical trial. $\mathrm{J}$ Thromb Haemost. 2008;6:622-31. [PubMed: 18208533]

13. Lexi-Drugs. Lexicomp. Hudson OH: Wolters Kluwer Health, In.; [Last accessed on 2016 Mar 01]. Factor IX Complex (Human) Available from: http://www.online.lexicom.

14. Huynh TK, Costello JL, Rebuck JA. Optimizing the dose of three-factor prothrombin complex concentrate in traumatic brain injury patients on warfarin therapy. Pharmacotherapy. 2014;34:260-4. [PubMed: 24338807] 


\section{Table 1}

Baseline Characteristics*

\begin{tabular}{lccc}
\hline & 4F-PCC $(n=57)$ & 3F-PCC $(n=77)$ & $P$ \\
\hline Age (years) & $57(14.7)$ & $74(10.8)$ & 0.0127 \\
Gender, male, $n(\%)$ & $29(50.9)$ & $43(55.8)$ & 0.325 \\
Weight $(\mathrm{kg})$ & $84.9(22.6)$ & $87.9(26.9)$ & 0.486
\end{tabular}

Anticoagulation

Indication

$\begin{array}{lcc}\text { Atrial fibrillation } & 29(50.1) & 53(70.1) \\ \text { DVT/PE } & 17(29.8) & 18(23.4) \\ \text { Heart valve } & 7(12.3) & 2(2.7) \\ \text { Other } & 4(7.0) & 2(2.7)\end{array}$

Bleed Location, n (\%)

Intracranial

23 (40)

Bowel

14 (24)

Chest

O (O)

Other

4 (7)

Multiple

$15(25)$

Missing

1 (2)

48 (62)

5 (7)

$4(6)$

$13(16)$

Baseline INR

$6.8(5.2)$

$15.1(7.0)^{\top}$

7 (9)

APACHE II

$16.2(7.9)^{ \pm}$

ISS

$14.4(5.9)^{\dagger}$

$18.4(9.2)^{=}$

${ }^{*}$ Continuous data presented as mean ( \pm SD), ${ }^{\dagger} 3 \mathrm{~F}-\mathrm{PCC}: \mathrm{n}=72 ; 4 \mathrm{~F}-\mathrm{PCC}: n=50$, 士3F-PCC : $n=72$; 4F-PCC: $n=50$ 


\section{Table 2}

INR Reversal Based on PCC Dose

\begin{tabular}{|c|c|c|}
\hline & $3 \mathrm{~F}-\mathrm{PCC}, n(\%)$ & 4F-PCC, $n(\%) *$ \\
\hline \multicolumn{3}{|l|}{20 units $/ \mathrm{kg}$} \\
\hline Baseline INR & 4.03 & 4.50 \\
\hline$\leq 1.3$ & $4 / 8(50)$ & $1 / 1(100)$ \\
\hline$\leq 1.5$ & $7 / 8(87.5)$ & $1 / 1(100)$ \\
\hline$\leq 1.7$ & $7 / 8(87.5)$ & $1 / 1(100)$ \\
\hline \multicolumn{3}{|l|}{$20-29.9$ units $/ \mathrm{kg}$} \\
\hline Baseline INR & 2.77 & 2.56 \\
\hline$\leq 1.3$ & $15 / 35(42.8)$ & $9 / 10(90)$ \\
\hline$\leq 1.5$ & $23 / 35(65.7)$ & $9 / 10(90)$ \\
\hline$\leq 1.7$ & $29 / 35(82.9)$ & $9 / 10(90)$ \\
\hline \multicolumn{3}{|l|}{$30-39.9$ units $/ \mathrm{kg}$} \\
\hline Baseline INR & 4.11 & 5.66 \\
\hline$\leq 1.3$ & $20 / 29(69.0)$ & $12 / 13(92.3)$ \\
\hline$\leq 1.5$ & $26 / 29(89.7)$ & $13 / 13(100)$ \\
\hline$\leq 1.7$ & $28 / 29(96.6)$ & $13 / 13(100)$ \\
\hline \multicolumn{3}{|c|}{$40-49.9$ units $/ \mathrm{kg}$} \\
\hline Baseline INR & 5.50 & 5.83 \\
\hline$\leq 1.3$ & $2 / 3(66.7)$ & $8 / 11(72.7)$ \\
\hline$\leq 1.5$ & $3 / 3(100)$ & $9 / 11(81.8)$ \\
\hline$\leq 1.7$ & $3 / 3(100)$ & $10 / 11(91)$ \\
\hline \multicolumn{3}{|l|}{$50-59.9$ units $/ \mathrm{kg}$} \\
\hline Baseline INR & 7.30 & 10.6 \\
\hline$\leq 1.3$ & $0 / 1(0)$ & $6 / 8(75)$ \\
\hline$\leq 1.5$ & $1 / 1(100)$ & $7 / 8$ (87.5) \\
\hline$\leq 1.7$ & $1 / 1(100)$ & $8 / 8(100)$ \\
\hline \multicolumn{3}{|l|}{$60-69.9$ units $/ \mathrm{kg}$} \\
\hline Baseline INR & 6.20 & 11.96 \\
\hline$\leq 1.3$ & $0 / 1(0)$ & $6 / 7(85.7)$ \\
\hline$\leq 1.5$ & $1 / 1(100)$ & $7 / 7(100)$ \\
\hline$\leq 1.7$ & $1 / 1(100)$ & $7 / 7(100)$ \\
\hline \multicolumn{3}{|c|}{$70-79.9$ units $/ \mathrm{kg}$} \\
\hline Baseline INR & -- & 11.23 \\
\hline$\leq 1.3$ & 0 & $3 / 3(100)$ \\
\hline$\leq 1.5$ & 0 & $3 / 3(100)$ \\
\hline$\leq 1.7$ & 0 & $3 / 3(100)$ \\
\hline
\end{tabular}

* 4 patients in 4F-PCC group did not have weight documented 